\title{
Sudden death Heart Pathology - A New Research Hipotesys in Drug Design strategy
}

\author{
Luisetto $\mathbf{M}^{1 *}$, Luca Cabianca ${ }^{2}$, Farhan $\mathrm{AK}^{3}$ and Ghulam $\mathbf{R M}^{4}$ \\ ${ }^{1}$ Department of Applied Pharmacologist, European specialist in lab medicine, Italy \\ ${ }^{2}$ Department of Biomedical, BiomedicalLaboratory \\ ${ }^{3}$ Department of Pharmacology, AIMSRC, India \\ ${ }^{4}$ Department of Medical \& Health Sciences, Peoples University of Medical \& Health Sciences for Woman, Pakistan
}

*Corresponding author: Luisetto M, European specialist in lab medicine, Applied Pharmacologist, Italy 29121 independent researcher

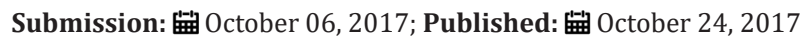

Keywords: Cardiology; Heart disease; Diagnostic systems

\section{Introduction}

We can see that heart is usually studied using many diagnostic strategies and under different Mechanism as functions, electric activity, receptorial, imaging, instrumental test, genetic profile, exercise tests, biopsy and so on. But what we can see is that until today in not possible to prevent some unexpected death. So we can think that perhaps we need more diagnostic test to right consider this aspect.

Can we use innovative methods to introduce a more useful system?

In ischemic condition some test can help to detect the residual heart capacity butcan we test in the same way the heart biochemicalmetabolic status or pharmacological profile? In a recent case we have see that in a transplant heart procedure was used an organ by a patient death due to cerebral damage after a heart attack and after few days the transplanted has exit us due by heart pathology. (Fact observed in an advanced healthcare system). It was reported that coronarography was normal in donor. Can we think a new method to early detect this kind of condition? Can we have a ranking useful to differentiate normal and abnormal patients?

How react a normal organ in para-physiological condition (alteration of electrolytes, acid-basebalace, oxygen level and so on).

In example as we easily see in other pathologies as diabetes or other endocrine disease patient are investigated in normal situation and after methabolic charge to correctly have a more complete information useful in clinical therapy. (The same in example to verify bioavability in-vivo in single transplanted patient under some immuno-depressant drugs are investigated with a kinetic emetic assay, or in neurology setting to correctly evaluate some anti epileptic pharmacological strategy Therapeutic drug monitoring is usually done to evaluate the single patient epatic status- citocrome ).

From biomedical literature we can see:

Buxton writed: "The guidelines are focused primarily on the appropriate use of implantable defibrillator. The bulk of the guidelines have very little basis in the underlying patho-physiology responsible for sudden cardiac death (SCD) in CHD patients" [1].

Shen et al. [2] showed that "The risk of SCD has changed over time among patients with symptomatic HF and reduced ejection fraction with sequential introduction of drugs as ACE inhibitors, angiotensin-receptor blockers, beta-blockers drugs, and mineralcorticoid-receptor antagonists" [2].

According Demosthenes G Katritsis et al. [3] "CAD is the most common cause of SCD, being responsible $80 \%$ of cases; cardiomyopathies and genetic channel-pathies account for most of the remainder. The incidence of SCD-related atherosclerotic CAD is 0.7 per 100,000 person-years in 18-35-year-olds, increasing to 13.7 per 100,000 in those $>35$ years of age. Valve disease and dilated or arrhythmo-genic cardio-myopathy are more common" [3].

Following IMA there is increased risk of SCD during the first months due to tachy-arrhythmias or other heart complications: reinfarction or myocardial rupture, and myocardial scar predisposes to monomorphic ventricular tachycardia (VT).Although most patients with a cardiac arrest have demonstrable CAD, however, less than half seem to have suffered an IMA.Only $38 \%$ of cardiac arrest survivors develop evidence of IMA. The most common causes of nonischaemic SCD are currently cardio-myopathy related to obesity 
alcoholism and fibrotic cardio-myopathy. In patients with preserved ejection fraction in the Cardiac Arrest Survivors with Preserved Ejection Fraction Registry, a etiological diagnosis was possible in approximately half of cardiac arrest survivors. A resuscitated cardiac arrest victim, preferably with documentation of VF, in whom known cardiac, respiratory, metabolic and toxicological a etiologies have been excluded through clinical evaluation is considered to have idiopathic VF. Several ion-channel and gene-coding mutations have been associated with idiopathic VF. Genetic testing diagnoses an inherited arrhythmia (genetic channelopathy) in up to $29 \%$ of families where a relative has died due to SCD. Several studies have also demonstrated a familial predisposition to SCD that may or may not be related to genetic channelopathies.

Coronary spasm is also a cause of cardiac arrest, particularly in male smokers with minimal or no pre-existing CAD. Mitral valve prolapse in female patients with ECG repolarisation abnormalities, and frequent complex ventricular ectopy, has also been associated with out-of-hospital cardiac arrest. An association between air pollution (fine particulate matter with an aerodynamic diameter $<2.5 \mu \mathrm{m}$ and ozone) and out-of-hospital cardiac arrest has recently been demonstrated. There is a circadian variation in SCD. The peak incidence of SCD occurs between 6 am and noon (and is blunted by beta-blockers), with a smaller peak occurring in the late afternoon for out-of-hospital VF arrests. The incidence is highest on Mondays.

In the young ( $<35$ years), the most common cause of SCD is arrhythmia, mostly in the context of an apparently normal heart. The most common causes of SCD are congenital abnormalities in those aged 0-13 years, primary arrhythmia in the 14-24-year age group, and CAD in those $>25$ years. In $5-20 \%$ of cases no significant cardiac abnormality is found at autopsy. In a recent Danish registry report on individuals aged $<50$ years, sudden death was caused by non-cardiac diseases, such as pulmonary embolism, meningitis and cerebrovascular bleeding, in $28 \%$ of cases.

In sports-related sudden death in the population, a clear diagnosis is made in $<25 \%$ of cases, but the cause is usually an acute coronary syndrome $(75 \%)$. In professional athletes, a diagnosis is usually made in up to $65 \%$ of cases and hypertrophic cardiomyopathy (HCM) is considered the main cause, at least in the United States, followed by arrhythmogenic right ventricular cardiomyopathy (ARVC, especially in the Veneto region of Italy), congenital coronary anomalies, genetic channelopathies, myocarditis, Wolff-ParkinsonWhite syndrome and Marfan syndrome, with blunt trauma, commotiocordis and heat stroke being less frequent causes. There is evidence, however, that HCM may not be the major cause of SCD in athletes. Autopsies in deceased NCAA athletes most often reveal a structurally normal heart $(25 \%)$, followed by coronary artery anomalies (11\%), myocarditis (9\%), ARVC (5\%) and aortic dissection (5\%), with HCM only demonstrated in $8 \%$ of individuals.

Findings from the Race Associated Cardiac Arrest Event Registry (RACER) indicate that marathons and half-marathons are associated with a low overall risk of cardiac arrest or sudden death $(1: 100,000)$, with deaths most commonly attributable to HCM $(26 \%)$ or atherosclerotic coronary disease (16\%). Some of these cardiac arrests might, however, have been provoked by heat stroke. CAD is the predominant cause of SCD in older athletes. Vigorous exertion can trigger cardiac arrest or SCD, especially in untrained persons, but habitual vigorous exercise diminishes the risk of sudden death during vigorous exertion. Most studies have found inverse associations between regular physical activity and SCD" [3]. So we have seen that varius etiologies are related to this pathology: Ventricular tachiaritmia, VF, bradiarithmia, electromechanical dissociation, asystole, pulseless electrical activity, channel pathologies y and other conditions. But in about 20\% cases not associated to abnormality found at autopsy, and that a Vigorous exertion can trigger a cardiac arrest or SCD, especially in untrained subjects [3].

\section{Discussion Conclusion}

Related to our consideration we can think a new method in heart disease staging strategy. New tests that can make possible to stress cardiac metabolism in normal, low and high working conditions or in para-phisio-pathological conditions but in local place (heart situation and not in plasma in example). We think that this new methods can be useful in much heart disease and condition to prevent some events. (Heart attacks, ischemic disease, artmia, heart failure, transplants, sports and so on). Every biomedical discipline has specific diagnostic discipline, but it can be useful to translate the various diagnostic system strategies from a discipline to other: this make possible to observe a phenomenon with a different point of view.

In the young common the most common causes of SCD IS arithmia mostly in apparently normal heart [3]. The difference in incidence SCD - atherosclerotic related between young patient and non young that we have seen is a fundamental objective data. The same also the relationships within sudden vigorous exertion untrained subjects (also in young) seem to show an inadequate methabolic response that must be adeguatelly investigate.

AS we have see in the citated references sudden death must be deeply studied in order to better clarify the pathologic cause [13] especially in young people. To prevent this kind of disease we think can be useful introduce new diagnostic strategy to verify in stressing conditions the local methabolic heart performance. This new methods can be the right instrument to produce helpful drug design strategies: a correct etiology and pathology information make possible to modulate better this kind of pathology.

\section{Clarifications}

This paper has not any diagnostic or therapy intent only to produce research hipotesys under strictly ethical principle in use.

\section{References}

1. Buxton AE (2017) Sudden death in ischemic heart disease. Int J Cardiol 237: 64-66.

2. Shen L, Jhund PS, Petrie MC, Claggett BL, Barlera S, et al. (2017) Declining Risk of Sudden Death in Heart Failure. N Engl J Med 377: 41-51.

3. Katritsis DG, Gersh BJ, John Camm A (2016) A Clinical Perspective on Sudden Cardiac Death. Arrhythm Electrophysiol Rev 5(3): 177-182. 